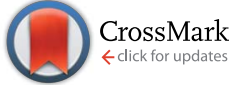

Cite this: J. Mater. Chem. A, 2017, 5, 11730

Received 23rd November 2016 Accepted 21st January 2017

DOI: 10.1039/c6ta10123b

rsc.li/materials-a

\section{Impact of synthetic routes on the structural and physical properties of butyl-1,4-diammonium lead iodide semiconductors $\uparrow$}

\author{
Majid Safdari, ${ }^{a}$ Dibya Phuyal, ${ }^{b}$ Bertrand Philippe, ${ }^{b}$ Per H. Svensson, ${ }^{\text {ae }}$ \\ Sergei M. Butorin, ${ }^{\mathrm{b}}$ Kristina O. Kvashnina, ${ }^{\text {cd }}$ Håkan Rensmo, ${ }^{\mathrm{b}}$ Lars Kloo ${ }^{\mathrm{a}}$ \\ and James M. Gardner*a
}

We report the significant role of synthetic routes and the importance of solvents in the synthesis of organicinorganic lead iodide materials. Through one route, the intercalation of dimethylformamide in the crystal structure was observed leading to a one-dimensional (1D) $\left[\mathrm{NH}_{3}\left(\mathrm{CH}_{2}\right)_{4} \mathrm{NH}_{3}\right] \mathrm{Pb}_{2} \mathrm{I}_{6}$ structure of the product. This product was compared with the two-dimensional (2D) $\left[\mathrm{NH}_{3}\left(\mathrm{CH}_{2}\right)_{4} \mathrm{NH}_{3}\right] \mathrm{Pbl}_{4}$ recovered from aqueous solvent based synthesis with the same precursors. UV-visible absorption spectroscopy showed a red-shift of $0.1 \mathrm{eV}$ for the band gap of the 1D network in relation to the 2D system. This shift primarily originates from a shift in the valence band edge as determined from photoelectron- and $\mathrm{X}$-ray spectroscopy results. These findings also suggest the iodide $5 p$ orbital as the principal component in the density of states in the valence band edge. Single crystal data show a change in the local coordination around iodide, while in both materials, lead atoms are surrounded by iodide atoms in octahedral units. The conductivity of the one-dimensional material $\left(\left[\mathrm{NH}_{3}\left(\mathrm{CH}_{2}\right)_{4} \mathrm{NH}_{3}\right] \mathrm{Pb}_{2} \mathrm{I}_{6}\right)$ was $50 \%$ of the twodimensional material $\left(\left[\mathrm{NH}_{3}\left(\mathrm{CH}_{2}\right)_{4} \mathrm{NH}_{3}\right] \mathrm{Pbl}_{4}\right)$. The fabricated solar cells reflect these changes in the chemical and electronic structure of both materials, although the total light conversion efficiencies of solar cells based on both products were similar.

\section{Introduction}

The growing interest in the application of organic-inorganic metal halides in optical devices has inspired the development of new materials. During recent years, the three-dimensional perovskite material methylammonium lead(II) iodide, (MA) $\mathrm{PbI}_{3}$, has been promoted as a light-absorber in photovoltaics. The perovskite (MA) $\mathrm{PbI}_{3}$ has a $\mathrm{Pm} 3 \mathrm{~m}$ space group containing a primitive cubic unit cell., ${ }^{1,2}$ Unlike many semiconductors, films of highly crystalline (MA) $\mathrm{PbI}_{3}$ may be generated through solution-based processes. In addition, $(\mathrm{MA}) \mathrm{PbI}_{3}$ has a direct band gap of $1.55 \mathrm{eV}$ and high extinction coefficients, which make this material an interesting candidate for solar cell applications. Although competing with existing solar cell technologies is

\footnotetext{
${ }^{a}$ Applied Physical Chemistry, Department of Chemistry, KTH Royal Institute of Technology, SE-10044, Stockholm, Sweden. E-mail: jgardner@kth.se; james.m. gardner@gmail.com

${ }^{b}$ Dept. of Physics and Astronomy, Uppsala Univ., Box 516, SE-75121, Uppsala, Sweden ${ }^{c}$ ESRF-The European Synchrotron, CS40220, 38043 Grenoble Cedex 9, France

${ }^{d}$ Helmholtz Zentrum Dresden-Rossendorf, Institute of Resource Ecology, 01314 Dresden, Germany

${ }^{e}$ SP Process Development, Forskargatan, 15121 Södertälje, Sweden

$\dagger$ Electronic supplementary information (ESI) available: Fig. S1-S9, Tables S1 and S2 including more detailed structural information, Tauc plot, detailed $I V$ data, $I V$ hysteresis, histogram of solar cell data. See DOI: 10.1039/c6ta10123b
}

challenging, researchers have reported exciting efficiencies up to $22.1 \%$ efficiency for solid-state solar cells based on methylammonium/formamidinium lead(II) iodide. ${ }^{3-8}$ The first reported use of (MA) $\mathrm{PbI}_{3}$ in solar cells was as a dye in liquid junction dyesensitized solar cells; ${ }^{3}$ however, the solar cells suffered from very low stability due to the high solubility of the methylammonium cation in the liquid electrolyte. Subsequently, the liquid electrolyte has been replaced with solid hole-transport materials (HTM), which have increased solar cell stability. ${ }^{4-8}$ However, atmospheric moisture causes decomposition of the perovskite on the time scale of hours due, primarily, to the hydrophilicity of the cations. ${ }^{\mathbf{9}, 10}$ It is the lack of the long-term stability of these perovskite films that impedes progress in the industrialization of these solar cells. ${ }^{11-13}$

While $\mathrm{Pb}^{2+}$ is known to be toxic and a risk to human health, a life cycle assessment study of (MA) $\mathrm{PbI}_{3}$ perovskite solar cells reported by Espinosa N. et al. ${ }^{\mathbf{1 4}}$ showed that the contribution of methylammonium iodide to human health risk was surprisingly far higher than the contribution from $\mathrm{Pb}$. Due to health concerns and instability issues, there is a clear need for alternatives to methylammonium lead iodide for use in solar cells. For substitution of the lead, the closest candidate would be tin. Several successful attempts at producing solar cells with Sn instead of $\mathrm{Pb}$ have yielded solar cell efficiencies of over $6 \% .^{15,16}$ Tin- and lead-based perovskites have very similar structural 
properties, which implies the importance of the structure of these materials with respect to their impressive physical properties. In our previous reports, we have reported relations between the structure of organic-inorganic lead halide materials and their physical properties., ${ }^{2,17}$ By changing organic cations to more stable cations, we correlate the change of dimensionality of these materials with their function as light absorbers in solid-state solar cells. ${ }^{2,17}$

The effect of synthetic routes on the three-dimensional (3D) methylammonium lead/tin iodide perovskite has been reported by Stoumpos C. C. et al., which shows a high dependency of the chemical and physical properties of the product on the synthetic routes. ${ }^{18}$ Furthermore, significant improvement in the efficiency has been achieved by the solvent engineering effect on the light absorber layer of the perovskite solar cells..$^{19,20}$ Our interest arose from these findings and our previous reports for further studies of these materials.

Herein, we report the strong effect of the synthetic route on the structure and properties of butyl-1,4-diammonium lead(II) iodide materials. Through two different synthetic routes, we have utilized butyl-1,4-diammonium iodide $\left[\mathrm{NH}_{3}\left(\mathrm{CH}_{2}\right)_{4} \mathrm{NH}_{3}\right] \mathrm{I}_{2}$ as a bication and anion source when mixed with lead iodide $\left(\mathrm{PbI}_{2}\right)$ to produce $\left[\mathrm{NH}_{3}\left(\mathrm{CH}_{2}\right)_{4} \mathrm{NH}_{3}\right] \mathrm{PbI}_{4}$ and $\left[\mathrm{NH}_{3}\left(\mathrm{CH}_{2}\right)_{4} \mathrm{NH}_{3}\right]$ $\mathrm{Pb}_{2} \mathrm{I}_{6}$. We investigated the structure of the products leading to two completely different structures: two-dimensional (2D) perovskite planes and one-dimensional (1D) non-perovskite chains. The structural differences of the crystals were confirmed by obtaining different X-ray powder diffraction patterns for the bulk material. These structural characterizations were followed by physical characterizations. Absorption spectra of the materials show a shift in the absorption band edge. The electronic structures of $\left[\mathrm{NH}_{3}\left(\mathrm{CH}_{2}\right)_{4} \mathrm{NH}_{3}\right] \mathrm{PbI}_{4}$ and $\left[\mathrm{NH}_{3}\left(\mathrm{CH}_{2}\right)_{4} \mathrm{NH}_{3}\right] \mathrm{Pb}_{2} \mathrm{I}_{6}$ were studied by synchrotron-based $\mathrm{X}$-ray spectroscopic methods. Solar cells were fabricated based on the two materials and utilized as light absorbers in solar cells; similar power conversion efficiencies of $1.1 \%$ were obtained for $\left[\mathrm{NH}_{3}\left(\mathrm{CH}_{2}\right)_{4}{ }^{-}\right.$ $\left.\mathrm{NH}_{3}\right] \mathrm{PbI}_{4} /(\mathrm{BdA}) \mathrm{PbI}_{4}$ based solid state solar cells and $\left[\mathrm{NH}_{3}\right.$ $\left.\left(\mathrm{CH}_{2}\right)_{4} \mathrm{NH}_{3}\right] \mathrm{Pb}_{2} \mathrm{I}_{6} /(\mathrm{BdA}) \mathrm{Pb}_{2} \mathrm{I}_{6}$ solar cells.

\section{Experimental section}

The precursors used in this work were all commercially available products and used without further purification. 1,4-Diaminobutane (99\%), lead(II) iodide (99.999\%), diethyl ether $(\geq 99.0 \%)$, and dimethylformamide (DMF) (purity $\geq 99 \%$ ) were purchased from Sigma-Aldrich. Spiro-OMeTAD (2,20,7,70-tet$\operatorname{rakis}[N, N$-di(4-methoxyphenyl)amino-9,9'-spirobifluorene $]$, purity $\geq 99.5$ HPLC) was purchased from Xi'an Polymer Light Technology Corp. Dyesol (18NRT) $\mathrm{TiO}_{2}$ paste was used and hydriodic acid was purchased from Alfa Aesar, 57\% w/w aqueous solution stabilized with $1.5 \%$ hypophosphorous acid.

\section{Synthesis}

For the synthesis of butyl-1,4-diammonium iodide, the previous procedure has been followed. ${ }^{17}$ Briefly, 1 mole equivalent of 1,4 diaminobutane (BA) $(0.003 \mathrm{M}, 0.264 \mathrm{~g})$ was mixed with 2 mole equivalents of $\mathrm{HI}(0.006 \mathrm{M}, 1.3703 \mathrm{~g})$ for 2 hours. $\left[\mathrm{NH}_{3}\left(\mathrm{CH}_{2}\right)_{4^{-}}\right.$ $\left.\mathrm{NH}_{3}\right] \mathrm{I}_{2} /(\mathrm{BdA}) \mathrm{I}_{2}$ was recovered by solvent evaporation and washed with diethyl ether (yield $89 \%$ ).

One-dimensional $\quad\left[\mathrm{NH}_{3}\left(\mathrm{CH}_{2}\right)_{4} \mathrm{NH}_{3}\right] \mathrm{Pb}_{2} \mathrm{I}_{6} /(\mathrm{BdA}) \mathrm{Pb}_{2} \mathrm{I}_{6} \quad$ was synthesized by mixing 1 mole equivalent of $(\mathrm{BdA}) \mathrm{I}_{2}(0.0015 \mathrm{M}$, $0.516 \mathrm{~g})$ with 2 mole equivalents of $\mathrm{PbI}_{2}(0.003 \mathrm{M}, 1.383 \mathrm{~g})$ in $\mathrm{DMF}$ $(5 \mathrm{~mL})$. Of the resulting solution, $1 \mathrm{~mL}$ was used for crystal growth with toluene as the anti-solvent. The remaining part of the solution was used to recover $(\mathrm{BdA}) \mathrm{Pb}_{2} \mathrm{I}_{6}$ powder by evaporation of the solvent at $50{ }^{\circ} \mathrm{C}$ using a rotary evaporator (yield $92 \%$ ).

The two-dimensional perovskite, $\left[\mathrm{NH}_{3}\left(\mathrm{CH}_{2}\right)_{4} \mathrm{NH}_{3}\right] \mathrm{PbI}_{4} /(\mathrm{BdA})$ $\mathrm{PbI}_{4}$, was synthesized according to the previous report. ${ }^{17}$ Briefly, 2 mole equivalents of $\mathrm{PbI}_{2}(0.003 \mathrm{M}, 1.383 \mathrm{~g})$ were dissolved in 4 $\mathrm{mL}$ HI solution. 1 mole equivalent of (BdA) $\mathrm{I}_{2}(0.0015 \mathrm{M}, 0.516 \mathrm{~g})$ was dissolved in $3 \mathrm{~mL}$ excess HI. The two solutions were mixed and stirred at $90{ }^{\circ} \mathrm{C}$ for 1 hour. In order to grow single crystals of the material, after half of the solvent is evaporated, the magnetic stir bar was taken out from the solution and the temperature was gradually decreased $\left(5{ }^{\circ} \mathrm{C} \mathrm{h} \mathrm{h}^{-1}\right)$ to $-10{ }^{\circ} \mathrm{C}$. Single crystals were obtained after keeping the solution at -10 ${ }^{\circ} \mathrm{C}$ for 24 hours. Crystals were recovered from the solution and used for crystallography. After removing the single crystals, the residual precipitate was washed with diethyl ether and used for further characterization (yield $81 \%$ ).

\section{Characterization}

Absorption spectroscopy. An approximately $\sim 250 \mathrm{~nm}$ thick mesoporous $\mathrm{TiO}_{2}$ film on a microscope slide was used for UVvisible spectroscopy. The $\mathrm{TiO}_{2}$ suspension (ratio of $1: 3.5: \mathrm{TiO}_{2}$ Dyesol paste : $99.5 \%$ ethanol) was spincoated onto a microscope slide followed by sintering at $500{ }^{\circ} \mathrm{C}$. A solution of $0.5 \mathrm{M}$ (BdA) $\mathrm{Pb}_{2} \mathrm{I}_{6}$ or (BdA) $\mathrm{PbI}_{4}$ in DMF was spincoated on the substrate followed by heating at $90{ }^{\circ} \mathrm{C}$ for $10 \mathrm{~min}$. UV-visible absorption measurements were performed using a UV-Visible Cary 300 spectrophotometer.

Single crystal X-ray crystallography. Single-crystal X-ray data were recorded on a Bruker APEX II diffractometer (Mo K $\alpha$ radiation), equipped with a CCD detector, at $200 \mathrm{~K}$. After selecting the appropriate crystals, they were mounted on a cryoloop using lowtemperature immersion oil and placed in a $\mathrm{N}_{2}$ cold stream. $\omega$ Scans and $\varphi$-scans were used for data recording, followed by integration with the Bruker SAINT ${ }^{21}$ software package. The absorption correction (Bruker SADABS) ${ }^{21}$ was based on fitting a function to the empirical transmission surface as sampled by multiple equivalent measurements. SHELXS and SHELX within the Bruker package were employed for solution and refinement of the crystal structures. ${ }^{21}$ Structure solution by direct methods resolved the positions of all atoms except hydrogens. The remaining non-hydrogen atoms were located by alternating cycles of least-squares refinements and difference Fourier maps. Hydrogen atoms were placed at calculated positions. The final refinements were performed with anisotropic thermal parameters for all non-hydrogen atoms.

X-ray powder diffraction. Before the experiment, the samples were ground to a fine powder with a mortar and pestle. Diffraction patterns of the fine powder samples were recorded 
by X-ray diffraction (PANalytical-X'Pert PRO diffractometer) using $\mathrm{Cu}-\mathrm{K} \alpha$ radiation. A $2 \theta$ range of $10^{\circ}$ to $40^{\circ}$ was used for scanning.

Conductivity. The measurements were carried out by the experimental methods presented in our previous studies. ${ }^{2,17}$ Briefly, a mesoporous $\mathrm{TiO}_{2}$ film (on a non-conductive substrate) was used as a substrate for spincoating the precursor solutions of the materials. After annealing at $90{ }^{\circ} \mathrm{C}$ for $10 \mathrm{~min}$, the silver back contact was evaporated. Over different bias voltages, the current response was collected under light illumination. We used the acquired slope of the current-voltage for conductivity calculation by eqn 1 .

$$
\sigma=\frac{W}{R L d}
$$

where $W$ is the width between silver back contact channels, $d$ is the total thickness of the material $/ \mathrm{TiO}_{2}$ film corrected for the $\mathrm{TiO}_{2}$ volume, and $L$ is the length of the $\mathrm{Ag}$ film. To correct for the $\mathrm{TiO}_{2}$ volume, the measured $d$ values were multiplied by $60 \%$ since the $\mathrm{TiO}_{2}$ films have $60 \%$ porosity.

Hard X-ray photoelectron spectroscopy (HAXPES). Hard Xray PES (HAXPES) was carried out at BESSY II (Helmholtz Zentrum Berlin) at the KMC-1 beamline using the HIKE endstation. ${ }^{22}$ A photon energy of $4000 \mathrm{eV}$ was selected using the first-order light from a Si (311) double-crystal monochromator (Oxford-Danfysik), and the photoelectron kinetic energies (KE) were measured using a Model R4000 analyzer (Scienta) optimized for high kinetic energies. A grazing angle was used for the measurement; the angle between the incident beam and the surface of the sample was $\sim 5^{\circ}$, i.e. normal emission to the entrance of the analyzer. The pressure in the analysis chamber was $\sim 10^{-8}$ mbar. The spectra presented were energy calibrated versus the Fermi level at zero binding energy, which was determined by measuring a gold plate in electrical contact with the sample and setting the Au $4 \mathrm{f} 7 / 2$ core level peak to $84.0 \mathrm{eV}$. The spectra were intensity calibrated using the most intense peak, if not stated otherwise. Intensity ratios presented between different core levels were calculated from the experimental results after correcting the intensity by the photoionization cross-section for each element, using database values. ${ }^{23}$

HERFD-XANES and RIXS. The HERFD-XANES and RIXS experiments were performed at beamline ID26 at the European Synchrotron Radiation Facility. ${ }^{24}$ The incident energy was selected using the Si (311) reflection from a double Si crystal monochromator. The energy calibration was performed using precursor $\mathrm{PbI}_{2}$. The HERFD-XANES spectra as well as the experimental procedure used to obtain them have been described previously. ${ }^{25}$ HERFD data at the $\mathrm{I}_{1} \mathrm{~L}_{1}$ edge were recorded at the maximum of the $\mathrm{L}_{\gamma 2,3}$ emission line $(E=5065$ $\mathrm{eV}$ ). RIXS spectra were recorded at the excitation energy of the first maximum of the iodine $\mathrm{L}_{1}$ edge in the energy range of 5173-5196 eV with a step size of $0.2 \mathrm{eV}$. The emitted energy in HERFD and RIXS was selected using the (331) reflection of three spherically bent Si crystal analyzers. The intensity was normalized to the incident flux measured using a photodiode. The spectrometer energy was calibrated using the elastic scattering line. The samples were positioned at $45^{\circ}$ to the beam. A helium- filled bag was used to reduce the absorption of fluorescence radiation between the cryostat and the spectrometer. All samples were checked for radiation damage, and no radiation damage could be detected within the acquisition time ( $5 \mathrm{~min}$ for HERFD and 25 min for RIXS) for the different spectra. Moreover, measurements were carried out on multiple spots on the samples.

Solar cell fabrication. Fluorine-doped tin-oxide (FTO) coated glass substrates (Pilkington TEC15) were washed for 30 minutes with detergent, ethanol and acetone in an ultrasonic bath respectively. The compact blocking layer was prepared on the FTO substrates by spray pyrolysis of a solution of $2 \mathrm{M}$ titanium isopropoxide in propanol at $500{ }^{\circ} \mathrm{C}$. To produce a mesoporous $\mathrm{TiO}_{2}$ film, a mixture of $1: 4.5 \mathrm{wt} \%$ ratio of $\mathrm{TiO}_{2}$ Dyesol paste with ethanol was used to spincoat a layer of $\mathrm{TiO}_{2}$ on top of the blocking layer at a rate of $4000 \mathrm{rpm}$. This was followed by sintering at $500{ }^{\circ} \mathrm{C}$ for $30 \mathrm{~min}$. A light absorbing layer was produced by spincoating $0.5 \mathrm{M}$ dimethylformamide (DMF; $\geq 99 \%$ ) precursor solutions of the materials on the $\mathrm{TiO}_{2}$ films and then the films were annealed at $90{ }^{\circ} \mathrm{C}$ for $10 \mathrm{~min}$.

A solution of $80 \mathrm{mM}$ SpiroOMeTAD, $200 \mathrm{mM}$ 4-tert-butylpyridine (TBP, 99\%) and $30 \mathrm{mM}$ bis(trifluoromethane) sulfonimide lithium salt (LiTFSI, 99\%, Io-li-tec) was prepared. The solvent for this solution was a mixture of $80 \mathrm{wt} \%$ chlorobenzene, $10 \mathrm{wt} \%$ acetonitrile (99.8\%), and $10 \mathrm{wt} \%$ 1,1,2,2-tetrachloroethane (TeCA). The hole transporting (HTM) layer was prepared through the spincoating of this solution at $2000 \mathrm{rpm}$ for 30 seconds. Finally, as the back contact, an 80-nm thick layer of gold was deposited by thermal evaporation. ${ }^{2,4,6,7}$ All of the fabrication steps (except the gold evaporation) were performed in an ambient environment (at $22{ }^{\circ} \mathrm{C}$ with a humidity of $46 \%$ as measured by using an AMPROBE TH-3 hygrometer).

\section{Solar cell characterization}

Current-voltage (IV). The characterization of solar cells was performed through the application of an external potential bias to the solar cell and recording the generated photocurrent with a Keithley model 2400 digital source meter. A $300 \mathrm{~W}$ collimated xenon lamp (Newport) was used as the light source. The light intensity was calibrated to $100 \mathrm{~mW} \mathrm{~cm}^{-2}$ under AM 1.5G solar light conditions by using a certified silicon solar cell (Fraunhofer ISE).

Incident photon-to-current efficiency (IPCE). A computercontrolled setup involving an assembly of a xenon lamp (Spectral Products ASB-XE-175), a monochromator (Spectral Products CM110) and a Keithley digital multimeter (Model 2700) was used for recording IPCE. A certified silicon solar cell (Fraunhofer ISE) was used for the calibration. A mask with an area of $0.126 \mathrm{~cm}^{2}$ (a circle with diameter $4 \mathrm{~mm}$ ) was applied for the measurements.

\section{Results and discussion}

\section{Material characterization and crystal growth}

The most popular and convenient route for the synthesis of the organic-inorganic lead(II) halide materials is through the 
solution methods. Through these methods, usually, lead halide will be mixed with the halide salt of organic cations/bications (see Fig. 1). ${ }^{5,26}$ A solvent will be added, and the synthesis will be done by heating and stirring this mixture for hours. To obtain the product, the influence of the solvent will be reduced by evaporation of the solvent or gradual cooling. This will generally result in the precipitation of the most thermodynamically stable product. This precipitation is normally governed by several important factors, e.g. (1) the Lewis acid and base activity of the components, (2) the iodide to lead ratio and (3) the tendency of the solvent to incorporate into the structure of the product. Diverse possible structures can be obtained for the products based on the cation/bication size, the ionic radius of the main metal, and the solvent activity. The dimensionality of the product can vary from zero-dimensional isolated units up to three-dimensional networks. This structural complexity provides a considerable opportunity for scientific research on material design and their properties. On the other hand, the reaction may result in an undesirable product, based on the diversity of the possible products.

The solvent effect is significant in determining the material structure and has been highlighted before. ${ }^{19}$ The great impact of the solvent on the perovskite deposition and architecture of the perovskite layer has been pointed out as a part of solar cell efficiency improvement. ${ }^{19,20}$ In the previous reports on the application of (MA) $\mathrm{PbI}_{3}$, the mixture of lead iodide with methyl ammonium iodide was stirred at $60{ }^{\circ} \mathrm{C}$ for 12 hours., ${ }^{\mathbf{4} 26}$ These reports have been followed by further developments in solar cell optimization that have lead to the use of mixed solvents, e.g. g-butyrolactone/ dimethylsulfoxide (GBL/DMSO) or dimethylformamide/dimethylsulfoxide (DMF/DMSO). These reports have proved that the crystallinity of the perovskite layer would be highly affected by the tendency of the solvent to evaporate versus to incorporate into the structure. ${ }^{15,24}$

\section{Single crystal data}

Through the single crystal characterizations, two different structures were obtained for these materials. For the product of Route $1(\mathrm{BdA}) \mathrm{Pb}_{2} \mathrm{I}_{6}$, a one-dimensional inorganic chain was obtained with a disordered intercalation of organic bications and solvent in between (see Fig. 2A). The inorganic chains in the $(\mathrm{BdA}) \mathrm{Pb}_{2} \mathrm{I}_{6}$ chemical structure consist of face-sharing lead iodide octahedral units. The space group of $P 1$ was found for the solvate-incorporated structure. The distance between the inorganic structural chains through space $(\mathrm{Pb}-\mathrm{Pb}$ distance) varies from $10.24 \AA$ to $13.04 \AA$. The oxygen atoms of the DMF are at a distance of $3.98 \AA$ to $6.2 \AA$ indicating a very weak bonding interaction with the inorganic chains. The intercalation of the solvent in the chemical structure of organic-inorganic metal halide materials is a known phenomenon, ${ }^{27}$ which has a significant impact on the physical properties of these materials. ${ }^{19,20}$ For the larger cations/bications, the distance between the inorganic structural units is longer, which accommodates larger vacancies between lead iodide chains that may be occupied by the solvent. The molecular volume of the (BdA) bication is substantially larger than the methylammonium cation and causes the connectivity of the inorganic units to be lost in one or more dimensions for the perovskite network. As well, bulkier cations/bications will increase the through-space distance between inorganic lead iodide units. This finding is consistent with our previous report, wherein the propylammonium lead(II) iodide structure incorporates gamma-butyrolactone due to the empty space between lead iodide chains. For the product of Route 2, a two-dimensional perovskite structure was produced. Details of this structure have been previously reported. ${ }^{17}$ Briefly, it can be mentioned here that $(\mathrm{BdA}) \mathrm{PbI}_{4}(001)$ structural planes were found to consist of octahedral corner sharing lead iodide planes and organic bications (BdA) as separating bridges between them (see Fig. 2B). The intra-chain $\mathrm{Pb}-\mathrm{Pb}$ distances in (BdA) $\mathrm{Pb}_{2} \mathrm{I}_{6}$ vary between $4.00 \AA$ and $4.07 \AA$. This can be compared with the same distance in the two-dimensional (BdA) $\mathrm{PbI}_{4}$ structure which is $6.08 \AA$ to $6.16 \AA$. Comparing $\mathrm{Pb}-\mathrm{I}-\mathrm{Pb}$ angles, for $(\mathrm{BdA}) \mathrm{Pb}_{2} \mathrm{I}_{6}$ the angles varied from 76.6 to 78.2 degrees while for $\mathrm{BdAPbI}_{4}$ they varied from 146.3 to 146.4 degrees. The changing $\mathrm{Pb}-\mathrm{I}-\mathrm{Pb}$ bond angles between (BdA) $\mathrm{Pb}_{2} \mathrm{I}_{6}$ and $(\mathrm{BdA}) \mathrm{PbI}_{4}$ suggest that the orbitals that contribute to the bonding of these materials may be different.

The structural differences between $(\mathrm{BdA}) \mathrm{Pb}_{2} \mathrm{I}_{6}$ and $(\mathrm{BdA}) \mathrm{PbI}_{4}$ were confirmed by X-ray powder diffraction on the recovered
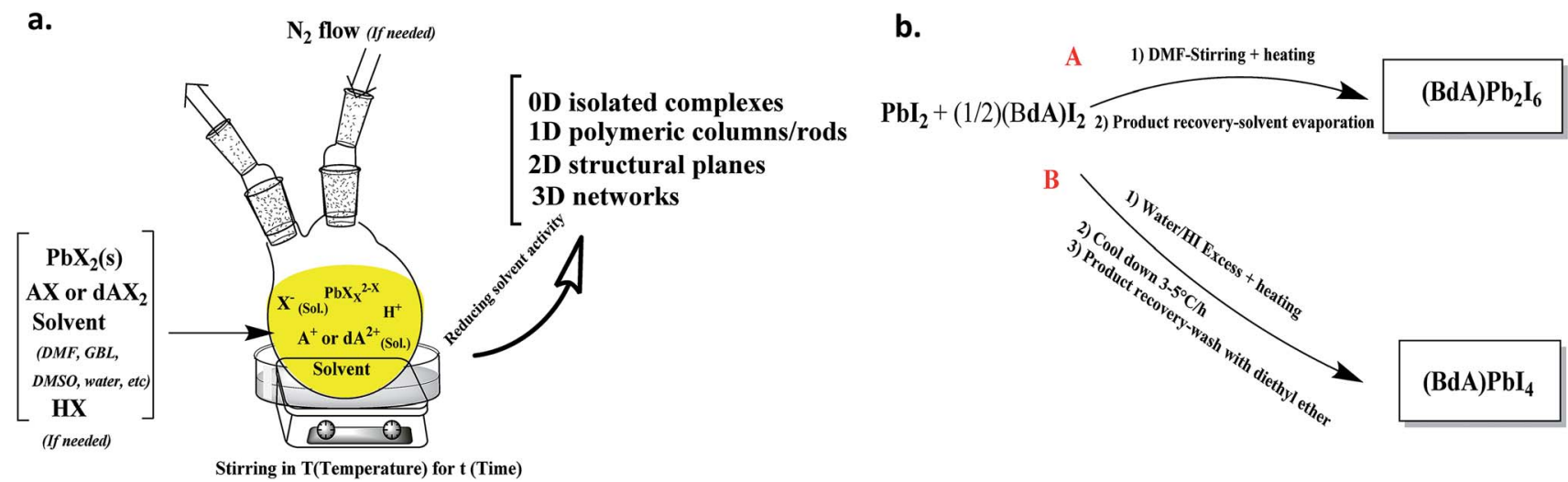

Fig. 1 General solution-based synthetic procedure for organic-inorganic lead halide materials, and (b) the synthetic routes for preparation of the $(\mathrm{BdA}) \mathrm{Pbl}_{4}$ and $(\mathrm{BdA}) \mathrm{Pb}_{2} \mathrm{I}_{6}$. 


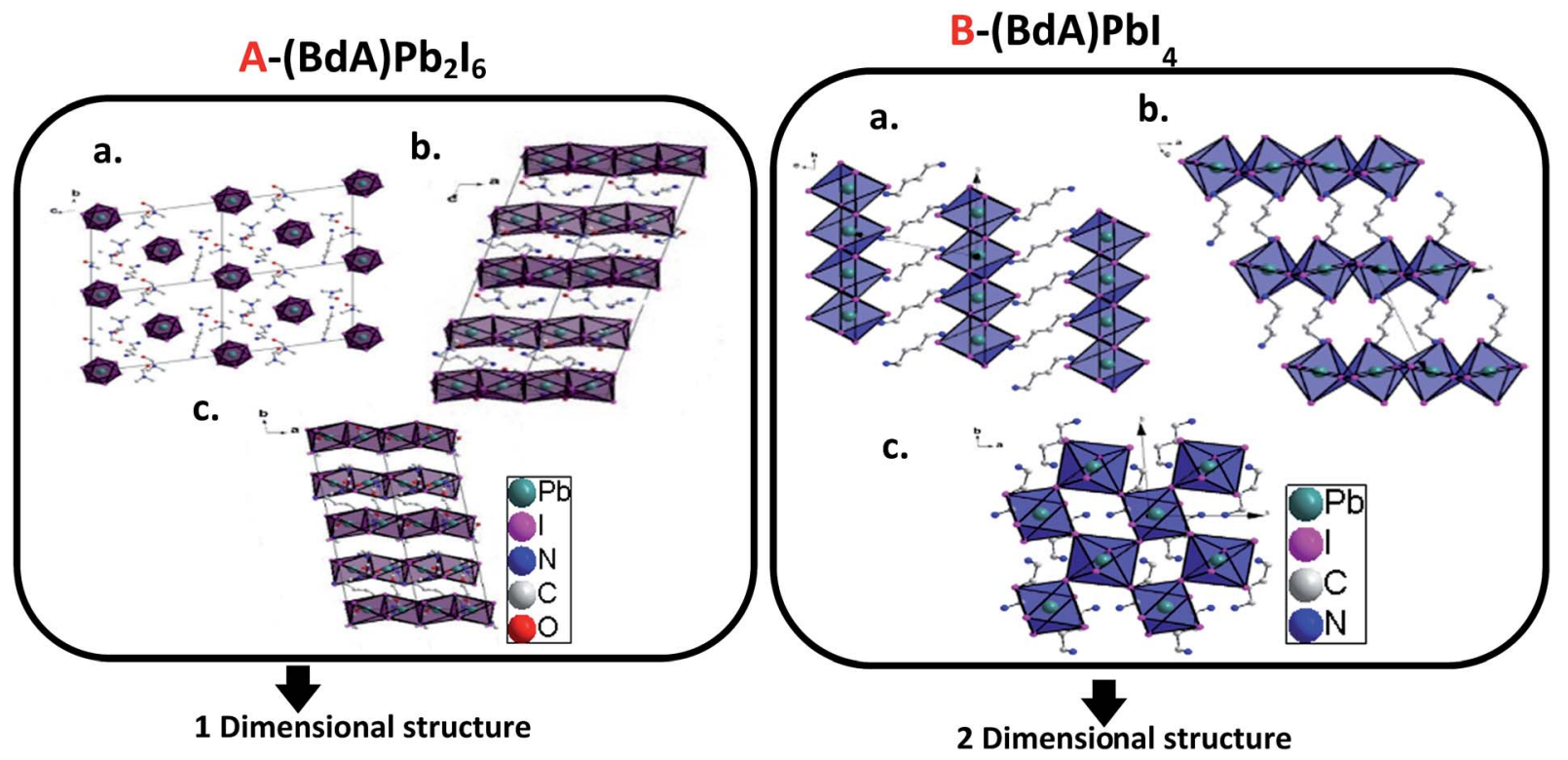

Fig. 2 Crystal structure of (A) solvated (BdA) $\mathrm{Pb}_{2} \mathrm{I}_{6}$ and (B) $(\mathrm{BdA}) \mathrm{Pbl}_{4}$ (ref. 17) along three axes.

powders (see Fig. 3). Different peaks were obtained for the 1D (BdA) $\mathrm{Pb}_{2} \mathrm{I}_{6}$ compared to the $2 \mathrm{D}(\mathrm{BdA}) \mathrm{PbI}_{4}$, illustrating structural changes in the bonding environment of the lead and iodide in each material. Several peaks were shifted to the lower angles for 1D compared to 2D. We attributed these peaks to the increasing volume of the unit cell for $1 \mathrm{D}(\mathrm{BdA}) \mathrm{Pb}_{2} \mathrm{I}_{6}\left(1968.27 \AA^{3}\right)$ as compared to $2 \mathrm{D}(\mathrm{BdA}) \mathrm{PbI}_{4}\left(765.28 \AA^{3}\right)$. The powder diffraction patterns show the poly-crystallinity of both samples although some broad peaks were recorded. These broad peaks can be due to some structural disorder in the powder samples. Similar phenomena were observed for previous two-dimensional materials. ${ }^{17}$ Shifts in the powder-XRD relative to the singlecrystal data for $(\mathrm{BdA}) \mathrm{Pb}_{2} \mathrm{I}_{6}$ suggest that the powdered material has a smaller unit volume than a single crystal. We attribute this to solvent loss from the powdered crystals of $(\mathrm{BdA}) \mathrm{Pb}_{2} \mathrm{I}_{6}$. The solvent is lost during the annealing process and results in a unit cell contraction (Fig. S7†).

\section{UV-vis absorption spectroscopy}

Characterization of absorption properties for the two different samples was performed and the obtained spectra are presented

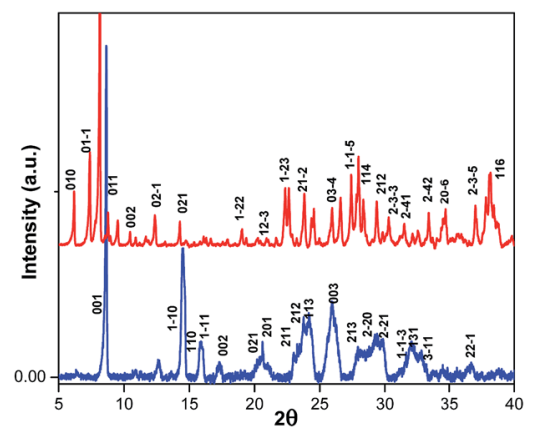

Fig. 3 Powder diffraction patterns for the --- $\left.(B d A) P\right|_{4}$ and --- $(B d A)$ $\mathrm{Pb}_{2} \mathrm{I}_{6}$. Miller indices were obtained from single crystal data. in Fig. 4. The first observed absorption peak was attributed to the band gap absorption between the valence and conduction bands. The direct band gap Tauc formula was used for the estimation of the band gap values of $(\mathrm{BdA}) \mathrm{Pb}_{2} \mathrm{I}_{6}$ and $(\mathrm{BdA})$ $\mathrm{PbI}_{4}{ }^{28} \mathrm{~A}$ red shift of $0.1 \mathrm{eV}$ in the absorption edge position was observed for the one-dimensional non-perovskite $(\mathrm{BdA}) \mathrm{Pb}_{2} \mathrm{I}_{6}$ when compared to the two-dimensional perovskite (BdA) $\mathrm{PbI}_{4}$. Extrapolation of the absorption edge from the Tauc plot (Fig. S8 $\dagger$ ) suggests band gap values of $2.28 \mathrm{eV}$ and $2.37 \mathrm{eV}$ for (BdA) $\mathrm{Pb}_{2} \mathrm{I}_{6}$ and (BdA) $\mathrm{PbI}_{4}$, respectively. Based on the intensity of the transition, the apparent absorption coefficient decreased for (BdA) $\mathrm{Pb}_{2} \mathrm{I}_{6}$ compared to (BdA) $\mathrm{PbI}_{4}$. A lower extinction coefficient for the solvated structure was observed for the methylammonium tin iodide perovskite ${ }^{27}$ and also for the formamidinium lead iodide perovskite. ${ }^{29}$ Based on most of the previous studies, ${ }^{2,27,30}$ the band gap is expected to be higher for one-dimensional materials compared to higher dimensionality systems. But in this particular study, the absorption study

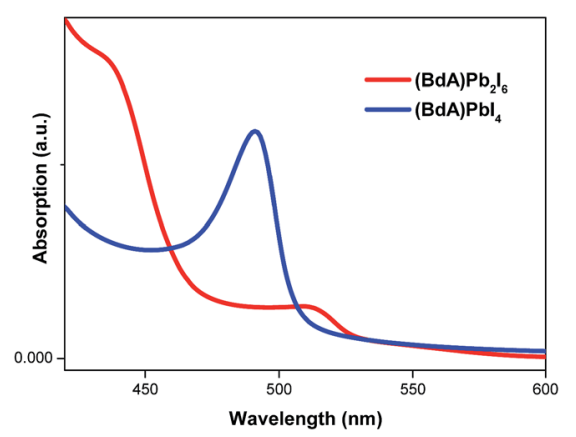

Fig. 4 Absorption spectra of spincoated --- 1D and --- 2D butyl-1,4diammonium lead(॥) iodide materials on $\mathrm{TiO}_{2}$ films. A small red shift in the band edge is observed for the one-dimensional $(\mathrm{BdA}) \mathrm{Pb}_{2} \mathrm{I}_{6}$ as compared to two-dimensional (BdA)Pbl, $544 \mathrm{~nm}$ and $523 \mathrm{~nm}$, respectively. 


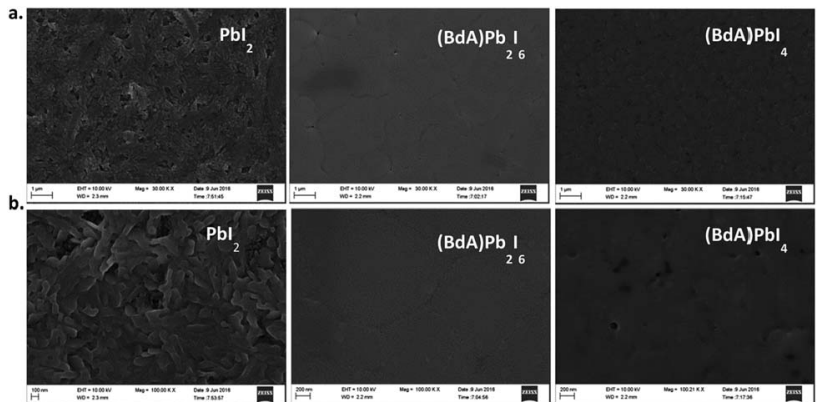

Fig. 5 Representative SEM images of annealed $\mathrm{Pbl}_{2},(\mathrm{BdA}) \mathrm{Pb}_{2} \mathrm{I}_{6}$, and ( $\mathrm{BdA}) \mathrm{Pbl}_{4}$ on mesoporous $\mathrm{TiO}_{2}$ at magnifications of (a) $30 \mathrm{KX}$, and (b) $100 \mathrm{KX}$. In both (BdA)Pb ${ }_{2} \mathrm{I}_{6}$ and $(\mathrm{BdA}) \mathrm{Pbl}_{4}$, compact, smooth, crystalline layers form.

showed rather small effects in the opposite direction. These interesting findings motivate us to analyze the electronic structure of these materials.

\section{Conductivity}

The conductivity of $(\mathrm{BdA}) \mathrm{Pb}_{2} \mathrm{I}_{6}$ was recorded as $5.3 \times 10^{-6} \mathrm{~S}$ $\mathrm{cm}^{-1}$ compared to $1.3 \times 10^{-5} \mathrm{~S} \mathrm{~cm}^{-1}$ for $(\mathrm{BdA}) \mathrm{PbI}_{4} \cdot{ }^{17} \mathrm{We}$ explain these data based on the chemical structure and dimensionalities of the two materials. In $(\mathrm{BdA}) \mathrm{PbI}_{4}$, charge conduction occurs through the lead iodide planes, while for (BdA) $\mathrm{Pb}_{2} \mathrm{I}_{6}$ connectivity and charge transport are solely along lead iodide chains. The finding supports previous evidence showing a high dependence of conductivity on the structural dimensionality. ${ }^{2}$

\section{X-ray based spectroscopy}

VB \& CB electronic structure. For X-ray and photoelectron spectroscopy characterizations, a thin layer of the butyldiammonium lead iodide material was spincoated onto a $\mathrm{TiO}_{2} / \mathrm{FTO}$ substrate. The SEM images of the representative samples are compared with $\mathrm{PbI}_{2} / \mathrm{TiO}_{2} / \mathrm{FTO}$ films in Fig. 5. 1D-(BdA)Pb $\mathrm{Pb}_{2} \mathrm{I}_{6}$ and 2D-(BdA) $\mathrm{PbI}_{4}$ films showed a uniform morphology. The experimental X-ray photoelectron spectroscopy (PES) valence levels are

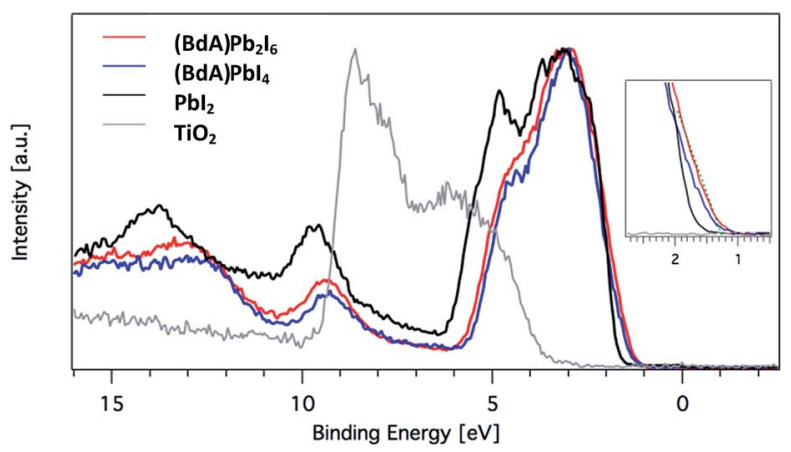

Fig. 6 Valence levels of the $\mathrm{TiO}_{2}$ film, $\mathrm{TiO}_{2} / \mathrm{Pbl}_{2}$ and $(\mathrm{BdA}) \mathrm{Pb}_{2} \mathrm{I}_{6}$ and $(\mathrm{BdA}) \mathrm{Pbl}_{4}$ measured with a photon energy of $4000 \mathrm{eV}$. A valence band edge shift was observed for --- $(B d A) \mathrm{Pb}_{2} I_{6}$ when compared to --- $(\mathrm{BdA})$ $\mathrm{Pbl}_{4}$.
Table 1 lodide to lead ratios obtained from the recorded intensities for core level photoelectrons as calculated from overview spectra

\begin{tabular}{lll}
\hline Sample & Chemical formula & I/Pb ratio \\
\hline$\left(\mathrm{BdA}^{2} \mathrm{~Pb}_{2} \mathrm{I}_{6}\right.$ & {$\left[\mathrm{NH}_{3}\left(\mathrm{CH}_{2}\right)_{4} \mathrm{NH}_{3}\right] \mathrm{Pb}_{2} \mathrm{I}_{6}$} & 2.65 \\
BdAPbI & {$\left[\mathrm{NH}_{3}\left(\mathrm{CH}_{2}\right)_{4} \mathrm{NH}_{3}\right] \mathrm{PbI}_{4}$} & 3.84 \\
Lead iodide & $\mathrm{PbI}_{2}$ & 1.78
\end{tabular}

shown in Fig. 6. The inset clearly indicates differences in the edge positions for the materials compared to $\mathrm{PbI}_{2}$. It also shows that the 1D and 2D films have rather similar spectral features with a small shift of $1 \mathrm{D}-(\mathrm{BdA}) \mathrm{Pb}_{2} \mathrm{I}_{6}$ towards smaller binding energies. In general, determining the exact band edge position ${ }^{31}$ relays different properties including the dispersion of the energy levels, but relative comparisons are often done by extrapolating the spectra with straight lines. The dashed green line indicates where the linearly extrapolated experimental spectra intersect with the baseline, a relative estimated measure of the valence band edge position versus the Fermi level. The intercept for the two samples is at a binding energy of 1.34 and $1.45 \mathrm{eV}$ for $(\mathrm{BdA}) \mathrm{Pb}_{2} \mathrm{I}_{6}$ and (BdA) $\mathrm{PbI}_{4}$, respectively, a small shift of about $0.1 \mathrm{eV}$. The extrapolated experimental spectra intersect is clearly shifted with respect to the $\mathrm{PbI}_{2}$ with about $1.69 \mathrm{eV}$. The main spectral features are observed around $2-6 \mathrm{eV}, 8-10 \mathrm{eV}$, and $12-14 \mathrm{eV}$. Although the samples were on a mesoporous $\mathrm{TiO}_{2}$ substrate film, we see very little contribution from the substrate with a valence edge position shifted almost $3 \mathrm{eV}$. Generally, the carbon and nitrogen in the organic bication are expected to contribute with some density of states at around 8 and $13 \mathrm{eV}$. The minor spectral contributions from these units come from low photoionization cross-sections of light elements at high photon energies.

An appropriate estimation of sample stoichiometry can be made by measuring intensities from the core levels of I $4 \mathrm{~d}$ and $\mathrm{Pb} 5 \mathrm{~d}$ (Fig. S2†). This estimation takes into account the differential atomic subshell photoionization cross-sections as well as comparison of similar orbitals. On the basis of these crosssection adjustments, we experimentally obtain appropriate relative intensities that are in accordance with what is expected from the stoichiometry of $\mathrm{PbI}_{2},(\mathrm{BdA}) \mathrm{Pb}_{2} \mathrm{I}_{6}$ and (BdA) $\mathrm{PbI}_{4}$ (see Table 1). For both (BdA) $\mathrm{Pb}_{2} \mathrm{I}_{6}$ and (BdA) $\mathrm{PbI}_{4}$, the magnitude of the $\mathrm{Pb} 4 \mathrm{f}$ core-level shifts is quite small $(0.05 \mathrm{eV})$; however, for I $3 \mathrm{~d}$, the core-level shift for (BdA) $\mathrm{Pb}_{2} \mathrm{I}_{6}$ and $(\mathrm{BdA}) \mathrm{PbI}_{4}$ is $0.12 \mathrm{eV}$. The experimental data give evidence that the positions of the valence positions shift largely due to the iodine $\mathrm{p}$ orbitals. The

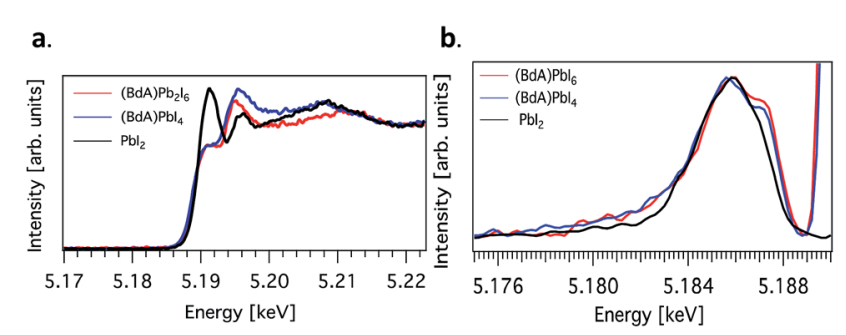

Fig. 7 (a) Plots of the unoccupied $n p$ states of iodine in the conduction band and (b) RIXS spectra for --- (BdA)Pb $\mathrm{I}_{6},---(\mathrm{BdA}) \mathrm{Pbl}_{4}$, and --- $\mathrm{Pbl}_{2}$. 


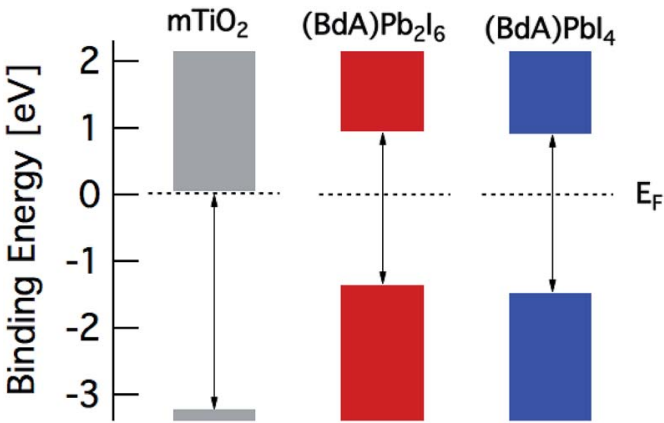

Fig. 8 Schematic diagram of the energy level positions versus the Fermi level. The relative valence band and conduction band positions (see text for details) are derived from band gap values based on the absorption spectra and PES data.

two butyldiammonium lead(II) iodide materials under study have geometrical configurations that are quite different from each other. The local bonding environment of iodine, which is connected by 1-dimensional face-sharing chains or 2-dimensional corner-sharing planes, indicates a distinct local arrangement that can explain the subtle differences in the electronic structure. This can be experimentally verified by probing iodine states specifically through absorption and emission spectroscopy.

Here, we explore the ability of high energy resolution fluorescence detection (HERFD) X-ray absorption near edge structure (XANES) and resonant inelastic X-ray scattering (RIXS) spectroscopy to distinguish between our similar but dimensionally varied samples. Fig. 7 shows the experimental HERFD-XANES spectra (left) and RIXS spectra (right) for this set. XANES probes the unoccupied density of states of the studied element. In our case $(\mathrm{BdA}) \mathrm{Pb}_{2} \mathrm{I}_{6}$ and (BdA) $\mathrm{PbI}_{4}$ clearly have different local geometries in the structure of the unoccupied iodine states. This indicates that there is a modification in the atomic position of neighbors, such as bond lengths and angles. This is entirely expected, since the local geometry of iodine is what sets these two samples apart in contrast to $\mathrm{Pb}$, which lies in a similar octahedral cage.

The iodide atoms have distinct bonding environments; therefore, one can expect to observe a different spectral character in the high energy XANES region. RIXS spectra are dominated by dipole-allowed transitions originating from occupied iodine $5 p$ orbitals, probing only occupied states, thus giving us a partial density of states near the VB edge. We see spectral features that are unique to our samples in contrast to $\mathrm{PbI}_{2}$, in particular a shoulder feature on the higher energy side. This is a consequence of structural changes due to iodine orbitals, which are involved in a different degree of hybridization with $\mathrm{Pb}$ 6s orbitals. The observed subtle shift to higher energies for (BdA) $\mathrm{Pb}_{2} \mathrm{I}_{6}$ in both HAXPES VB and iodine RIXS indicates that the observed change in the electronic structure of the valence band is primarily due to the iodine orbitals.

The band diagram of the materials obtained from spectroscopic data is presented in Fig. 8. The valence band edge is obtained from PES data with the Fermi level set to $0 \mathrm{eV}$ in accordance with the Au Fermi level. The position of the conduction band minimum (CBM) relative to $E_{\mathrm{F}}$ is estimated by subtracting the optical band gap from the valence band maximum (VBM). From this picture, a suitable combination of our materials can be utilized in many different device architectures for efficient charge extraction.

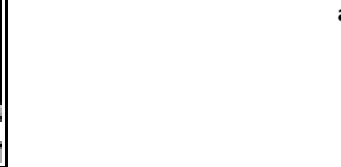

a.

b.
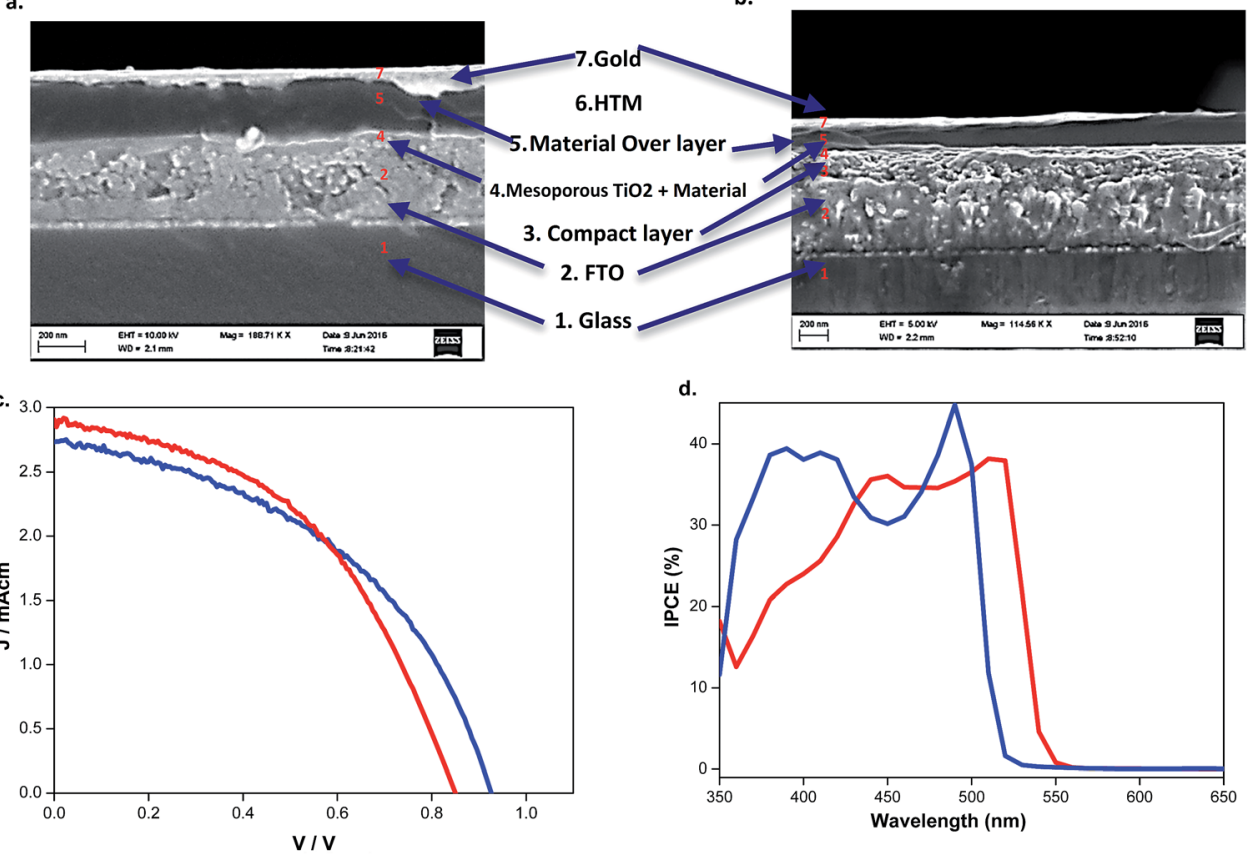

d.

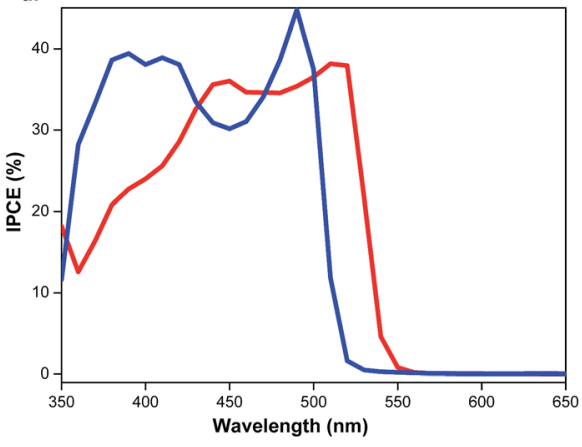

Fig. $9 \mathrm{SEM}$ cross-sectional image of (a) $(\mathrm{BdA}) \mathrm{Pb}_{2} \mathrm{l}_{6}$ and (b) $(\mathrm{BdA}) \mathrm{Pbl}_{4}$ based solid-state solar cells showing their related architectures. Obtained (c) current-voltage and (d) IPCE curves of --- $(\mathrm{BdA}) \mathrm{Pb}_{2} \mathrm{I}_{6}$ and --- $(\mathrm{BdA}) \mathrm{Pbl}_{4}$ solar cells. A mesoporous $\mathrm{TiO}_{2}$ layer was used as the electron transporting material and gold was used as the back contact. 
Table 2 Photovoltaic performance of solar cells based on (BdA)Pbl 4 and $(B d A) \mathrm{Pb}_{2} I_{6} . I V$ measurements were performed under simulated 1 sun AM 1.5G illumination

\begin{tabular}{|c|c|c|c|c|c|c|c|}
\hline $\mathrm{BdAPbI}_{4}$ & 1.1 & 925 & 2.73 & 0.45 & 36 & 2.37 & $1.3 \times 10^{-5}$ \\
\hline
\end{tabular}

Solar cells. As the final step for the characterizations, we applied these two materials as light absorbing layers in the solar cells. Solar cells were made with a conventional structure as shown in Fig. 9a and b. A thin layer of the materials was formed on top of the mesoporous $\mathrm{TiO}_{2}$ layer, after spincoating the $0.5 \mathrm{M}$ DMF precursor solution. Solar cells were characterized and the obtained photovoltaic performances are presented in Table 2 . Identical efficiencies were obtained for both materials, 1.1\% power conversion efficiency, despite having vastly different structures and electronic properties. 10 solar cells were fabricated based on each material which have shown good reproducibility (the detailed $I V$ data and the related efficiency histogram are presented in Tables S1 and S2 and Fig. S9†). Despite very similar light conversion efficiency, different voltage and current values were obtained. The small shift for the current density can be attributed to the lower band gap of $\sim 0.1$ $\mathrm{eV}$ for $(\mathrm{BdA}) \mathrm{Pb}_{2} \mathrm{I}_{6}$ compared to that of $(\mathrm{BdA}) \mathrm{PbI}_{4}$. The difference in the $V_{\text {oc }}$ between the two materials can be explained by the changes in the chemical and electronic structure (see Fig. 1 and 8). For $(\mathrm{BdA}) \mathrm{Pb}_{2} \mathrm{I}_{6}$, one-dimensional chains lead to lower conductivity than for $(\mathrm{BdA}) \mathrm{PbI}_{4}$. However, this does not appear to lead to a substantial increase in electron recombination since APCE values are nearly identical. $I V$ current data were recorded at $10 \mathrm{mV} \mathrm{s}^{-1}$ scan rate to avoid hysteresis. ${ }^{32}$ Solar cells utilizing (BdA) $\mathrm{Pb}_{2} \mathrm{I}_{6}$ were shown to have negligible hysteresis (Fig. S6 $\dagger$ ); similar data were observed for (BdA)PbI ${ }_{4} \cdot{ }^{17}$ Recorded IPCE curves are well-matched with the absorption spectra, verifying the small red shift in the band edge for $(\mathrm{BdA}) \mathrm{Pb}_{2} \mathrm{I}_{6}$.

\section{Conclusion}

The effects of solvents and synthetic routes of butyldiammonium lead iodide were investigated through the characterization of the two products. Chemical and electronic structures were studied in detail. Dimethylformamide showed a tendency to intercalate into the crystal structure and change the chemical structure of the final product to a one-dimensional structure. The impacts of the structural changes on the physical properties were deeply studied. A small red shift for the band edge was observed for one-dimensional (BdA) $\mathrm{Pb}_{2} \mathrm{I}_{6}$ compared to twodimensional (BdA) $\mathrm{PbI}_{4}$. This shift in the band edge was confirmed by IPCE measurements on the solar cells using the two materials as light absorbing layers. Through X-ray based spectroscopies, it was determined that the shift in the band gap mostly originates from changes in the valence band. HERFDXANES and XPS studies suggest that for both materials the dominant contribution to the valence band comes from the iodine $5 \mathrm{p}$ orbital while the lead $6 \mathrm{~s}$ orbital is the primary contribution to the conduction band. Through the transmission of the chemical structure from two-dimensional to onedimensional, the chemical environment and bonding of iodide atoms will be mostly altered while lead atoms remain in the center of octahedral units with minor changes in the distance to the iodide corners. The HERFD-XANES and XPS findings confirm these structural changes by showing a large change in the valence band edge position compared to small changes in conduction band edges. The conductivity of the one-dimensional material, $(\mathrm{BdA}) \mathrm{Pb}_{2} \mathrm{I}_{6}$, was half that of the two-dimensional material, $(\mathrm{BdA}) \mathrm{PbI}_{4}$. The decrease in conductivity indicates the importance of connectivity for maintaining electron conductivity throughout the material. Coincidentally, the fabricated solar cells based on the two materials showed similar efficiencies, despite the substantial differences in the chemical structure and absorption properties that affect the recorded voltages and current densities.

\section{Acknowledgements}

This work was supported by the Swedish Government through "STandUP for ENERGY", the Swedish Energy Agency, the Swedish Research Council, Knut \& Alice Wallenberg Foundation, and the National Research Foundation of Korea (NRF2014K1A3A1A47067328).

\section{References}

1 T. Baikie, Y. Fang, J. M. Kadro, M. Schreyer, F. Wei, S. G. Mhaisalkar, M. Graetzel and T. J. White, J. Mater. Chem. A, 2013, 1, 5628-5641.

2 M. Safdari, A. Fischer, B. Xu, L. Kloo and J. M. Gardner, J. Mater. Chem. A, 2015, 3, 9201-9207.

3 A. Kojima, K. Teshima, Y. Shirai and T. Miyasaka, J. Am. Chem. Soc., 2009, 131, 6050-6051.

4 H.-S. Kim, C.-R. Lee, J.-H. Im, K.-B. Lee, T. Moehl, A. Marchioro, S.-J. Moon, R. Humphry-Baker, J.-H. Yum, J. E. Moser, M. Gratzel and N.-G. Park, Sci. Rep., 2012, 2, 591.

5 M. M. Lee, J. Teuscher, T. Miyasaka, T. N. Murakami and H. J. Snaith, Science, 2012, 338, 643-647.

6 J. Burschka, N. Pellet, S.-J. Moon, R. Humphry-Baker, P. Gao, M. K. Nazeeruddin and M. Gratzel, Nature, 2013, 499, 316319.

7 W. S. Yang, J. H. Noh, N. J. Jeon, Y. C. Kim, S. Ryu, J. Seo and S. I. Seok, Science, 2015, 348, 1234-1237.

8 http://www.nrel.gov/ncpv/images/efficiency_chart.jpg. 
9 J. Yang, B. D. Siempelkamp, D. Liu and T. L. Kelly, ACS Nano, 2015, 9, 1955-1963.

10 S. Pathak, A. Sepe, A. Sadhanala, F. Deschler, A. Haghighirad, N. Sakai, K. C. Goedel, S. D. Stranks, N. Noel, M. Price, S. Hüttner, N. A. Hawkins, R. H. Friend, U. Steiner and H. J. Snaith, ACS Nano, 2015, 9, 2311-2320.

11 T. A. Berhe, W.-N. Su, C.-H. Chen, C.-J. Pan, J.-H. Cheng, H.-M. Chen, M.-C. Tsai, L.-Y. Chen, A. A. Dubale and B.-J. Hwang, Energy Environ. Sci., 2016, 9, 323-356.

12 X. Li, M. Ibrahim Dar, C. Yi, J. Luo, M. Tschumi, S. M. Zakeeruddin, M. K. Nazeeruddin, H. Han and M. Grätzel, Nat. Chem., 2015, 7, 703-711.

13 D. Wang, M. Wright, N. K. Elumalai and A. Uddin, Sol. Energy Mater. Sol. Cells, 2016, 147, 255-275.

14 N. Espinosa, L. Serrano-Luján, A. Urbina and F. C. Krebs, Sol. Energy Mater. Sol. Cells, 2015, 137, 303-310.

15 N. K. Noel, S. D. Stranks, A. Abate, C. Wehrenfennig, S. Guarnera, A.-A. Haghighirad, A. Sadhanala, G. E. Eperon, S. K. Pathak, M. B. Johnston, A. Petrozza, L. M. Herz and H. J. Snaith, Energy Environ. Sci., 2014, 7, 3061-3068.

16 F. Hao, C. C. Stoumpos, D. H. Cao, R. P. H. Chang and M. G. Kanatzidis, Nat. Photonics, 2014, 8, 489-494.

17 M. Safdari, P. H. Svensson, M. T. Hoang, I. Oh, L. Kloo and J. M. Gardner, J. Mater. Chem. A, 2016, 4(40), 15638-15646.

18 C. C. Stoumpos, C. D. Malliakas and M. G. Kanatzidis, Inorg. Chem., 2013, 52, 9019-9038.

19 N. J. Jeon, J. H. Noh, Y. C. Kim, W. S. Yang, S. Ryu and S. I. Seok, Nat. Mater., 2014, 13, 897-903.

20 Y. Rong, Z. Tang, Y. Zhao, X. Zhong, S. Venkatesan, H. Graham, M. Patton, Y. Jing, A. M. Guloy and Y. Yao, Nanoscale, 2015, 7, 10595-10599.
21 B. A. B. S. Apex II, Bruker AXS Inc., Madison, WI, USA, 2013. 22 M. Gorgoi, S. Svensson, F. Schäfers, G. Öhrwall, M. Mertin, P. Bressler, O. Karis, H. Siegbahn, A. Sandell, H. Rensmo, W. Doherty, C. Jung, W. Braun and W. Eberhardt, Nuclear Instruments and Methods in Physics Research Section A: Accelerators, Spectrometers, Detectors and Associated Equipment, 2009, 601, 48-53.

23 J. Scofield, J. Electron Spectrosc. Relat. Phenom., 1976, 8, 129137.

24 C. Gauthier, V. A. Sole, R. Signorato, J. Goulon and E. Moguiline, J. Synchrotron Radiat., 1999, 6, 164-166.

25 A. J. Atkins, C. R. Jacob and M. Bauer, Chem.-Eur. J., 2012, 18, 7021-7025.

26 J.-H. Im, C.-R. Lee, J.-W. Lee, S.-W. Park and N.-G. Park, Nanoscale, 2011, 3, 4088-4093.

27 F. Hao, C. C. Stoumpos, P. Guo, N. Zhou, T. J. Marks, R. P. H. Chang and M. G. Kanatzidis, J. Am. Chem. Soc., 2015, 137, 11445-11452.

28 J. Tauc, R. Grigorovici and A. Vancu, Phys. Status Solidi B, 1996, 15, 10.

29 T. M. Koh, K. Fu, Y. Fang, S. Chen, T. C. Sum, N. Mathews, S. G. Mhaisalkar, P. P. Boix and T. Baikie, J. Phys. Chem. C, 2014, 118, 16458-16462.

30 D. B. Mitzi, in Progress in Inorganic Chemistry, John Wiley \& Sons, Inc., 2007, pp. 1-121.

31 J. Endres, D. A. Egger, M. Kulbak, R. A. Kerner, L. Zhao, S. H. Silver, G. Hodes, B. P. Rand, D. Cahen, L. Kronik and A. Kahn, J. Phys. Chem. Lett., 2016, 7, 2722-2729.

32 E. L. Unger, E. T. Hoke, C. D. Bailie, W. H. Nguyen, A. R. Bowring, T. Heumuller, M. G. Christoforo and M. D. McGehee, Energy Environ. Sci., 2014, 7, 3690-3698. 